\title{
IL-29 Exhibits Anti-Tumor Effect on Pan-48 Pancreatic Cancer Cells by Up-regulation of P21 and Bax
}

\author{
DEAN BALABANOV ${ }^{1 *}$, LEI ZHAO ${ }^{2 *}$, ZIWEN ZHU $^{3}$, ZACHARY E. HUNZEKER $^{3}$, HANNAH M. TONNER $^{3}$, \\ VIVI A. DING ${ }^{1}$, MARK R. WAKEFIELD ${ }^{3}$, QIAN BAI ${ }^{3}$ and YUJIANG FANG ${ }^{1,3}$ \\ ${ }^{1}$ Department of Microbiology \& Immunology, Des Moines University, Des Moines, IA, U.S.A.; \\ ${ }^{2}$ Department of Respiratory Medicine, the 2nd People's Hospital of Hefei and \\ Hefei Hospital Affiliated to Anhui Medical University, Hefei, P.R. China; \\ ${ }^{3}$ Department of Surgery, University of Missouri School of Medicine, Columbia, MO, U.S.A.
}

\begin{abstract}
Background/Aim: Pancreatic cancer is the most lethal cancer of the digestive system. IL-29 is a new member of the IFN family and well-known for its strong antiviral activity. However, its direct effect on pancreatic cancer is still unclear. This study was performed to investigate if IL-29 has any direct effect on Pan-48 pancreatic cancer cells. Materials and Methods: Clonogenic survival assay, cell proliferation, and caspase-3 activity kits were used to evaluate the effects of IL-29 on cell survival, proliferation, and apoptosis of Pan-48 pancreatic cancer cells. RT-PCR and IHC were subsequently performed to explore IL-29's potential molecular mechanisms. Results: The percentage of colonies of Pan-48 cells was decreased following the addition of IL-29. This was consistent with a decreased optical density $(O D)$ value of cancer cells. Furthermore, the relative caspase-3 activity in cancer cells was increased after the addition of IL-29, indicating increased apoptosis of cancer cells. The anti-proliferative effect of IL-29 on cancer cells correlated with increased expression of the anti-proliferative molecule p21. The pro-apoptotic effect of IL-29 on cancer cells correlated with an increased expression of the pro-apoptotic molecule Bax. Conclusion: IL-29 constrains Pan-48 pancreatic cell growth via up-regulation of p21 and Bax. Our study suggests a potential use of IL-29 in immunotherapy for pancreatic cancer treatment.
\end{abstract}

*These Authors contributed equally to this study.

Correspondence to: Yujiang Fang, Department of Microbiology \& Immunology, Des Moines University, 3200 Grand Avenue, IA 50312, U.S.A. Tel: +1 5152711435, Fax: +1 5152711543, e-mail: yujiang.fang@dmu.edu

Key Words: IL-29, pancreatic cancer, p21, bax, proliferation, apoptosis.
Pancreatic cancer (PCA) is estimated to have cost over 44,000 lives in 2018, as it is the most lethal digestive cancer (1). Trailing only lung, colorectal, and breast cancer, pancreatic cancer has the highest mortality rate in the world. The 5-year survival rate for people with stage IA, IB, IIA, III, and IV pancreatic cancer is $14 \%, 12 \%, 7 \%, 3 \%$, and $1 \%$ respectively. What concerns both physicians and patients is that PCA is considered a "silent" cancer, as the pancreas cannot be palpated on physical exam and its tumor serum markers are often non-specific. Most closely related to PCA are tumor markers CA19-9 and the carcinoembryonic antigen (CEA). However, these protein markers do not always rise in pancreatic cancer patients, and even if they do, the cancer is likely to have already metastasized to a distant location (1). Moreover, these tumor markers can be elevated even if the patient does not have pancreatic cancer. Thus, physicians do not routinely use blood tests to screen for PCA, as they are nonspecific in nature. Furthermore, screening for average-risk patients is not currently recommended by any major professional group, as the benefits of screening do not outweigh the risks of going unscreened. Thus, PCA is a frightening and deadly diagnosis.

Current treatment for PCA revolves around the individual patients' life expectancy and goals. Several options are present for patients who wish to battle PCA, including surgery, ablation, radiation, and chemotherapy. However, as the previously mentioned 5-year survival rates indicate, current treatments are not adequate. Cytokine therapy is a relatively new treatment strategy for cancer patients. The role of cytokines in tumor biology is currently undergoing intense study as these peptides influence tumor growth, invasion, angiogenesis, and metastasis (2). The activation of the immune system for the therapeutic benefit in cancer patients has been previously investigated and the administration of cytokines has shown to be a successful cancer treatment that, however, warrants further investigation $(3,4)$. 

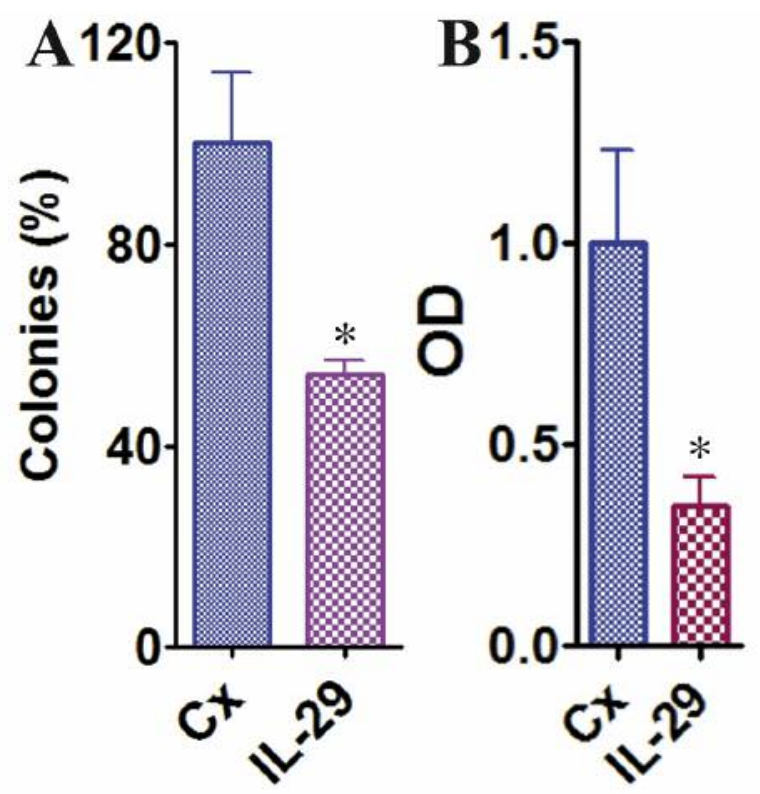

Figure 1. Effect of IL-29 on growth and proliferation on Pan-48 cells. (A) Clonogenic survival assay of Pan-48 pancreatic cancer cells in the presence or absence of $I L-29(50 \mathrm{ng} / \mathrm{mL})$. The number of colonies was counted and expressed as a percentage of the total colonies compared to the control ( $C x$, without IL-29). (B) Cell proliferation was evaluated with a proliferation kit in the presence and absence of IL-29. Results are expressed as the mean optical density $(O D+S E M$ in each group. A significant difference in the percentage of colonies and $O D$ value in each group compared to that of controls is indicated by an asterisk $(p<0.05)$.

In 2003 , a new member of the IFN $\lambda$ family was discovered, named IL-29 (5). Well-known for its strong antiviral activity, IL-29's mechanism of action is by targeting epithelial cells. Thus, high levels of IL-29 are expected during infections of the gastrointestinal (GI), respiratory tract, and mucosal regions (5). In recent years, the role of IL29 in the pathogenesis of an assortment of cancers has been investigated. IL-29 has been found to either induce tumor promoting effects or tumor inhibiting effects, depending on the cancer cell type (5). Concomitant with its ambiguous role in other types of cancer, the role of IL-29 in pancreatic cancer is also very unclear. This current study was performed to investigate if IL-29 has any direct effect on the widely investigated Pan-48 pancreatic cancer cells.

\section{Materials and Methods}

Human pancreatic cell line. The human Pan-48 PCA cell line used in this study was provided by Dr. Citrin from the Radiation Oncology Branch in NIH (Center for Cancer Research, National Cancer Institute, MD, USA). Pan-48 PCA cells were cultured in DMEM (Invitrogen, Carlsbad, CA, USA), supplemented with 10\% heatinactivated FBS and 1\% penicillin-streptomycin (Invitrogen) as previously described (6-9). Treatment with cytokine IL-29 or medium alone was performed when Pan- 48 cells reached $70 \%$ confluence.
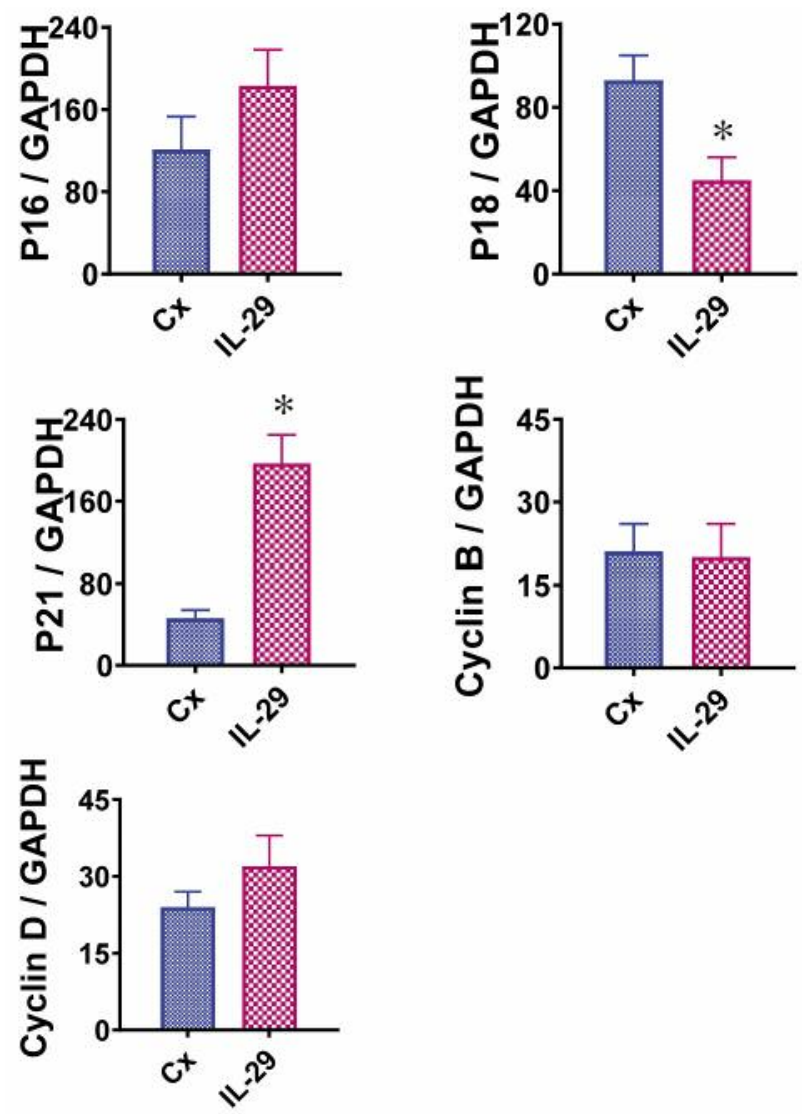

Figure 2. Effect of IL-29 on the expression of pro- and anti-proliferative molecules in Pan-48 cells as evaluated by RT-PCR. Results are expressed as the mean ratio of molecule densitometric units/GAPDH+SEM, and are representative of 3 independent experiments. An asterisk indicates a significant difference $(p<0.05)$ between untreated $(C x)$ and IL-29-treated groups.

Treatment of pancreatic cell line with IL-29. Pan-48 cells were treated with IL-29 at the concentration of $50 \mathrm{ng} / \mathrm{mL}$ (Shenandoah Biotechnology, Warwick, PA, USA) for 3 days or DMEM medium alone. The concentration and incubation time for the IL-29 treatment was based on our studies (6-13).

Clonogenic survival assay. Three days after incubation with IL-29, Pan-48 cells were counted in a hemocytometer following detachment. Clonogenic survival assay was performed as described previously (6-9). The number of colonies was counted and then expressed as a percentage of total colonies in controls.

Determination of proliferation with Quick Cell Proliferation Assay Kit. A Quick Cell Proliferation Assay Kit (BioVision, Milpitas, CA, USA) was used to evaluate Pan-48 cell proliferation as previously described (6-9).

RT-PCR. Pan-48 cells treated with and without IL-29 were homogenized in TRIzol (Invitrogen). RNA was extracted and its concentration was determined using NanoDrop (Invitrogen). 


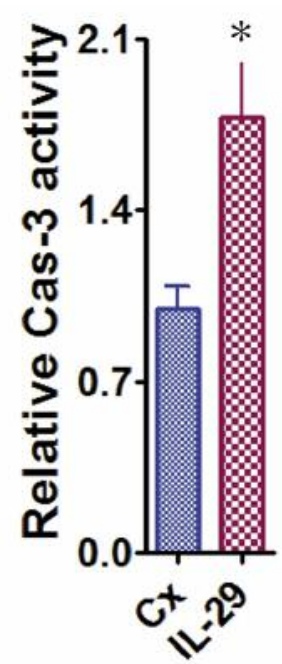

Figure 3. Effect of IL-29 on the apoptosis of Pan-48 Cells. Cellular caspase-3 activity was measured using the caspase-3/CPP32 colorimetric assay kit. Results are expressed as mean activity relative to controls $(R T)+S E M$. Assays were done in triplicates. An asterisk indicates a significant difference $(p<0.05)$ between untreated $(C x)$ and IL-29-treated groups.
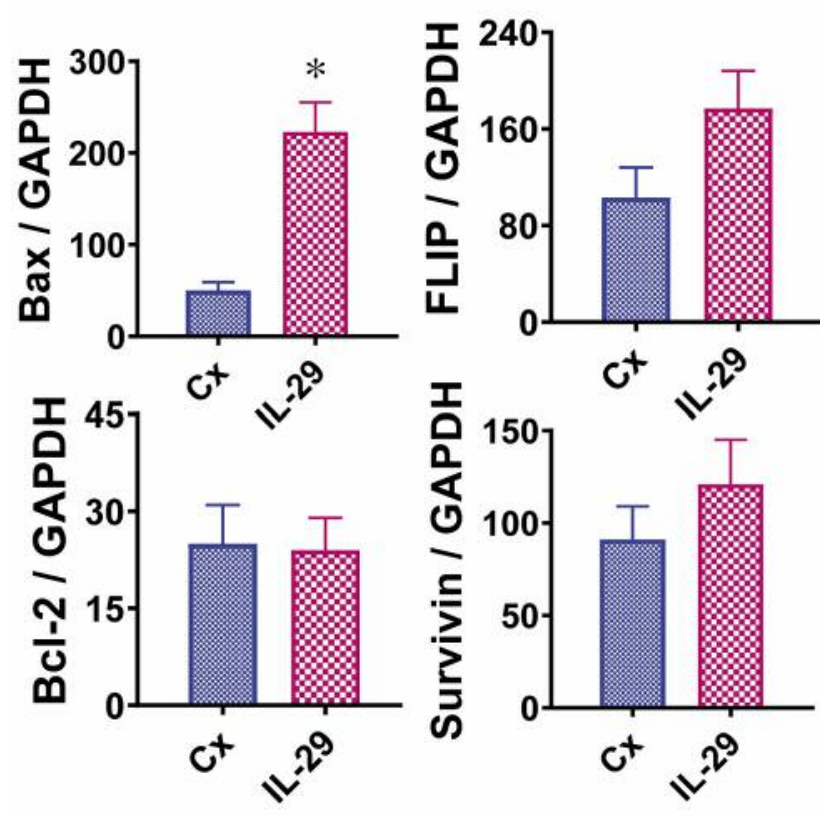

Figure 4. Effect of IL-29 on the expression of pro- and anti-apoptotic molecules in Pan-48 cells evaluated by RT-PCR. Results are expressed as the mean ratio of molecule densitometric units. An asterisk indicates a significant difference $(p<0.05)$ between untreated $(C x)$ and IL-29treated groups.

Reverse transcription was performed with $1 \mu \mathrm{g}$ of Pan-48 RNA, as previously described (7-11). GAPDH was used to monitor and ensure that an equal amount of RNA was amplified. Sequences for primers used in this study have been described previously (6-9).

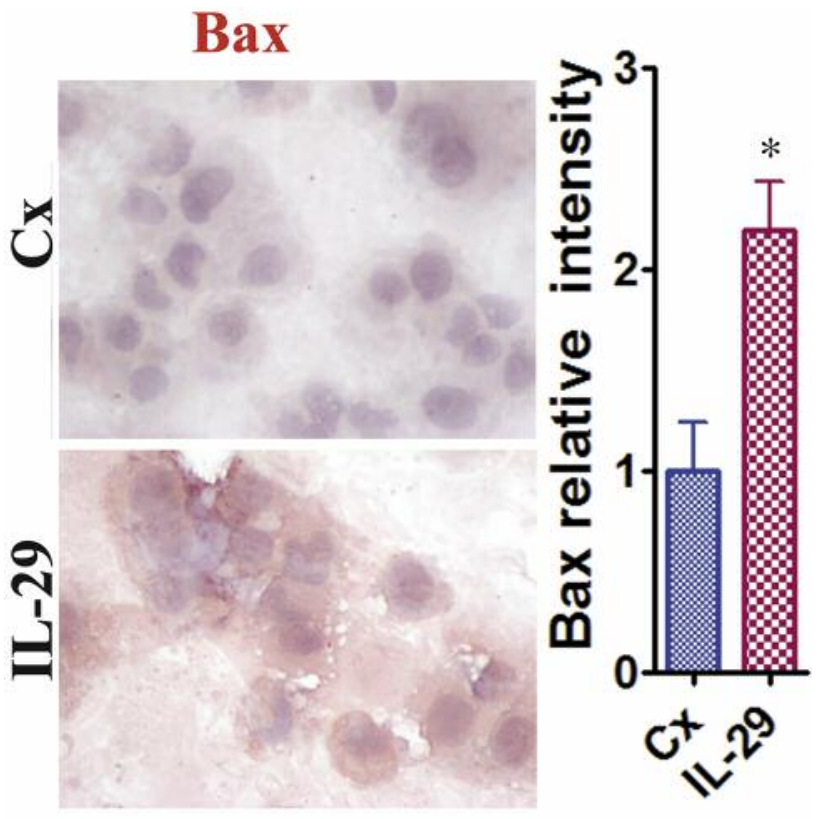

Figure 5. Effect of IL-29 on the expression of Bax in Pan-48 cells evaluated by immunohistochemistry. Cytoplasmic BAx staining is obvious in the IL-29-treated group (bottom image, original magnification: 400$)$ compared to the nuclear staining in the untrated (Cx) group (top image, original magnification: x400). Bax staining intensity was analyzed and results are expressed as the average integrated staining intensity relative to that of control $(\mathrm{Cx})$ cells (graph). A significant difference in staining intensity in the IL-29-treated group compared to the control is indicated by an asterisk $(p<0.05)$.

Measurement of Caspase-3 activity. A caspase-3/CPP32 colorimetric assay kit (BioVision) was further used to evaluate apoptosis by measuring the cellular caspase-3 activity and the results were expressed as a relative caspase- 3 activity compared to that of control untreated cells (6-9).

Immunohistochemistry (IHC). IHC staining for Bax was performed as previously described (6-9). Following incubation with $0.1 \%$ saponin in $1 \%$ BSA for 0.5 hour, cancer cell slides were incubated with a specific polyclonal Antibody (Ab) (Santa Cruz Biotechnology, Santa Cruz, CA, USA) for 1 hour at room temperature. Immunoreactivity was demonstrated using the avidinbiotin complex immunoperoxidase system (Vector, Burlingame, CA, USA) and was developed by using NovaRED (Vector) as the chromogen following incubation with a secondary biotinylated $\mathrm{Ab}$ for 0.5 hour (Jackson, West Grove, PA, USA). Hematoxylin was used as a negative control and for counterstaining. Average immunostaining intensity for a specific protein was measured with the assistance of MetaMorph image analysis software. Results are expressed as the average integrated immunostaining intensity of 3 slides + SEM relative to that in control.

Statistics. All experiments were repeated at least two to three times. Statistical analysis was carried out using an unpaired two-tailed Student's $t$-test. A $p$-Value $<0.05$ was considered significant. 


\section{Results}

IL-29 inhibits growth and proliferation of Pan-48 cells. Pan48 cells were used to investigate the effect of IL-29 on PCA. Overall, the percentage of colonies of Pan-48 cells significantly decreased following treatment with IL-29. This is further supported by the decreased optical density (OD) value following treatment with IL-29 (Figure 1).

IL-29 up-regulates the mRNA expression of the antiproliferative molecule $p 21$. The normal balance that exists between pro- and anti- proliferative molecules is important for cancer cell proliferation and survival. The molecules p16, p18, and p21 have all been shown to bear important anti-proliferative properties. In contrast, cyclin B and cyclin D have demonstrated important pro-proliferative effects. Using RTPCR, the mRNA expression of these pro- and anti-proliferative molecules in Pan-48 cells treated with or without IL-29 (50 $\mathrm{ng} / \mathrm{mL}$ ) was evaluated (Figure 2). Overall, an increased mRNA expression of the anti-proliferative molecule p21, was observed. These results indicate that a decrease in Pan-48 cell proliferation correlates with an increased expression of p21 as induced by IL-29. Interestingly, a decreased mRNA expression of the anti-proliferative molecule p18 was also observed; however, the detailed reason remains unknown.

IL-29 promotes apoptosis of Pan-48 cells. The inhibitory effect of IL-29 on the growth of Pan- 48 cells could be in part due to a mechanism that acts via increased apoptosis. To investigate the pro-apoptotic effect of IL-29 on Pan- 48 cells, $70 \%$ confluent Pan-48 cells were treated with IL-29 or medium alone for 3 days and apoptosis was subsequently evaluated using a caspase3 activity kit. Overall, a significant increase in apoptosis was found in the Pan-48 cells treated with IL-29 (Figure 3). This result supports a second mechanism of action for IL-29, which is the inhibition of Pan-48 cells growth by inducing apoptosis.

IL-29 up-regulates the expression of the pro-apoptotic molecule Bax. In order to test the molecular mechanism of action by which IL-29 induces apoptosis in Pan-48 cells, the mRNA expression of the major pro- and anti- apoptotic molecules, such as Bax, FLIP, Bcl-2 and survivin, was measured using RT-PCR. Overall, mRNA expression of Bax was significantly increased in the Pan-48 cells treated with IL-29 as compared to the control (Figure 4). Results obtained from IHC staining further confirmed our findings (Figure 5). These results indicate that an increase in apoptosis of Pan-48 cells correlates with an increased expression of Bax induced by IL-29.

\section{Discussion}

In this study, we demonstrated that IL-29 exhibits an antitumoral effect on Pan-48 PCA cells by inhibiting their proliferation and promoting their apoptosis. The inhibitory effect of IL-29 on proliferation is associated with the upregulation of the anti-proliferative molecule $\mathrm{p} 21$, while the apoptosis inductive effect of IL-29 is associated with the upregulation of the pro-apoptotic molecule Bax. These results implicate IL-29 as a novel and promising potential therapeutic agent for patients with PCA.

In recent years, cytokine-cancer immunotherapy has gained extensive interest in research (22). As the mortality and incidence of PCA continues to rise, more patients continue to present with advanced-staged disease that is virtually untreatable when diagnosed. While current treatment strategies have been unremarkable in the treatment for PCA, novel cytokine therapies represent hope for patients diagnosed with this deadly disease. Further exploration of the various effects of the existing cytokines on cancer is needed. This area of research has the potential to become the mainstay in treatment not only against pancreatic cancer, but to help many different cancer patients around the world. Despite our promising result is not consistent with a previously done study by Torres et al., which has demonstrated that up-regulation of serum IL-29 can be one of several cytokines that carries a poor prognosis in patients with pancreatic ductal adenocarcinoma (14). It should be noted that in this study there was no assessment of the molecular mechanism of action and that treatment with IL-29 was not specific to Pan-48 cells, which likely explains the differences in outcomes between their results and ours. Interestingly, our data are consistent with a study from Novak et al., which has shown that IL-29 has an inhibitory effect on the growth of multiple myeloma cancer cells (15). Further research is needed to fully elucidate the effects of IL-29 on various cell lines so that its potential power in cytokine therapy can be fully harnessed.

Though the recent studies investigating the role of IL-29 as a potential cancer immunotherapy molecule are encouraging, our study is the first to demonstrate potential molecular mechanisms of action of this cytokine. In our study, an upregulation of $\mathrm{p} 21$ represented a significant anti-proliferative potential when PCA cells were treated with IL-29. p21 is a cyclin-dependent kinase inhibitor that is primarily associated with the inhibition of CDK2 (cyclin-dependent kinase 2), which leads to cell cycle arrest in the setting of DNA damage (16-18).

Bax is a member of the Bcl-2 gene family and is a regulator of apoptosis (19-21). Bax has been shown to be involved in the p53-mediated pathway of apoptosis, which involves the release of cytochrome $\mathrm{c}(9,10)$. Through the regulation of apoptosis, Bax is a crucial protein involved in the regulation of cancer cells. Thus, our finding of increased mRNA expression of Bax is critical for the inhibitory mechanism of IL-29 on PCA, as it represents an increase on the levels of apoptosis in cancer cells.

Interestingly, a decreased mRNA expression of the antiproliferative molecule p18 was also observed in our 
experiment. Although the exact reason and mechanism for this decrease is yet unknown, we believe that a decreased mRNA expression of p18 might be a compensatory phenomenon arising from the inhibition of PCA by IL-29. If this is true, the PCA would seem to be fighting off the inhibitory effects of IL-29. Interestingly, this is not the first time we have noticed p18 in such a role. Our lab has observed on previous occasions a similar decrease in p18 mRNA expression and we therefore believe it to be due to IL-29's inhibition in PCA cells $(6,8,22)$.

This study was limited due to the examination of the effects of IL-29 in a single pancreatic cell line. However, future studies should include the examination of the effects of IL-29 across multiple cell lines within a specific type of cancer. This way, the effects of IL-29 can be fully elucidated and its role in cancer cytokine therapy can be examined more closely. Overall, it has become clear that the effects of IL29 are cancer cell-specific. Future research must also be directed towards investigating of what other cancer cells IL29 may be inhibiting or stimulating. This way, a working knowledge of cancer types, which could potentially be treated by IL-29, can be established.

Conclusively, this study represents a promising start and a hope that cytokine immunotherapy, and IL-29 specifically, can one day be the future of pancreatic cancer therapy for patients around the world.

\section{Conflicts of Interest}

The Authors declare no conflicts of interest.

\section{Authors' Contributions}

YF conceived and designed this study. All Authors performed experiments. YF and ZZ analyzed and interpreted the data. DB and YF wrote the draft and made critical revision. All Authors approved the final version of the article.

\section{Acknowledgements}

This study was supported by grants for Yujiang Fang (Iowa Science Foundation Grant ISF 16-8, IOER 05-14-01, IOER 112-3749 and IOER 112-3104).

\section{References}

1 Siegel RL, Miller KD and Jemal A: Cancer statistics, 2018. CA Cancer J Clin 68(1): 7-30, 2018. PMID: 29313949. DOI: $10.3322 /$ caac. 21442

2 Matsuo Y, Takeyama $\mathrm{H}$ and Guha S: Cytokine network: New targeted therapy for pancreatic cancer. Curr Pharm Des 18(17): 2416-2419, 2012. PMID: 22372505. DOI: 10.2174/1381612 8112092416

3 Vincent A, Herman J, Schulick R, Hruban RH and Goggins M: Pancreatic cancer. Lancet 378(9791): 607-620, 2011. PMID: 21620466. DOI: 10.1016/S0140-6736(10)62307-0
4 Porta C, Paglino C, Imarisio I and Bonomi L: Cytokine-based immunotherapy for advanced kidney cancer: Past results and future perspectives in the era of molecularly targeted agents. ScientificWorldJournal 7: 837-849, 2007. PMID: 17619768. DOI: $10.1100 /$ tsw.2007.154

5 Kelm NE, Zhu Z, Ding VA, Xiao H, Wakefield MR, Bai Q and Fang Y: The role of il-29 in immunity and cancer. Crit Rev Oncol Hematol 106: 91-98, 2016. PMID: 27637354. DOI: 10.1016/j.critrevonc.2016.08.002

6 Fang Y, Chen X, Bai Q, Qin C, Mohamud AO, Zhu Z, Ball TW, Ruth CM, Newcomer DR and Herrick EJ: Il-9 inhibits htb-72 melanoma cell growth through upregulation of p21 and trail. J Surg Oncol 111(8): 969-974, 2015. PMID: 25988864. DOI: $10.1002 /$ jso. 23930

7 Fang Y, Zhao L, Xiao H, Cook KM, Bai Q, Herrick EJ, Chen X, Qin C, Zhu Z and Wakefield MR: Il-33 acts as a foe to mia paca2 pancreatic cancer. Med Oncol 34(2): 23, 2017. PMID: 28058630. DOI: 10.1007/s12032-016-0880-3

8 Nicholl MB, Chen X, Qin C, Bai Q, Zhu Z, Davis MR and Fang Y: Il-32 $\alpha$ has differential effects on proliferation and apoptosis of human melanoma cell lines. J Surg Oncol 113(4): 364-369, 2016. PMID: 27100023. DOI: 10.1002/jso.24142

9 Zhu Z, Davidson KT, Brittingham A, Wakefield MR, Bai Q, Xiao $\mathrm{H}$ and Fang Y: Trichomonas vaginalis: A possible foe to prostate cancer. Med Oncol 33(10): 115, 2016. PMID: 27613161. DOI: 10.1007/s12032-016-0832-y

10 Fang Y, Sharp GC, Yagita H and Braley-Mullen H: A critical role for trail in resolution of granulomatous experimental autoimmune thyroiditis. J Pathol 216(4): 505-513, 2008. PMID: 18810759. DOI: $10.1002 /$ path.2428

11 Fang Y, Wei Y, Demarco V, Chen K, Sharp GC and BraleyMullen H: Murine flip transgene expressed on thyroid epithelial cells promotes resolution of granulomatous experimental autoimmune thyroiditis in dba/1 mice. Am J Pathol 170(3): 875887, 2007. PMID: 17322373. DOI: 10.2353/ajpath.2007.060816

12 Fang Y, Yu S and Braley-Mullen H: Tgf- $\beta$ promotes proliferation of thyroid epithelial cells in ifn- $\gamma-/-$ mice by down-regulation of p21 and p27 via akt pathway. Am J Pathol 180(2): 650-660, 2012. PMID: 22119715. DOI: 10.1016/j.ajpath.2011.10.009

13 Manning AA, Zhao L, Zhu Z, Xiao H, Redington CG, Ding VA, Stewart-Hester T, Bai Q, Dunlap J, Wakefield MR and Fang Y: Il-39 acts as a friend to pancreatic cancer. Med Oncol 36(1): 12, 2018. PMID: 30506430. DOI: 10.1007/s12032-0181236-y

14 Torres C, Linares A, Alejandre MJ, Palomino-Morales RJ, Caba O, Prados J, Aranega A, Delgado JR, Irigoyen A, MartinezGalan J, Ortuno FM, Rojas I and Perales S: Prognosis relevance of serum cytokines in pancreatic cancer. Biomed Res Int 2015: 518284, 2015. PMID: 26346854. DOI: $10.1155 / 2015 / 518284$

15 Novak A, Grote D, Ziesmer S, Rajkumar V, Doyle S and Ansell $\mathrm{S}$ : A role for ifn- $\lambda 1$ in multiple myeloma b cell growth. Leukemia 22(12): 2240, 2008. PMID: 18830264. DOI: 10.1038/ leu. 2008.263

16 Johnson DG and Walker CL: Cyclins and cell cycle checkpoints. Annu Rev Pharmacol Toxicol 39: 295-312, 1999. PMID: 10331086. DOI: 10.1146/annurev.pharmtox.39.1.295

17 Zhang D, Li X, Chen C, Li Y, Zhao L, Jing Y, Liu W, Wang X, Zhang Y, Xia H, Chang Y, Gao X, Yan J and Ying $\mathrm{H}$ : Attenuation of p38-mediated mir-1/133 expression facilitates myoblast proliferation during the early stage of muscle 
regeneration. PLoS One 7(7): e41478, 2012. PMID: 22911796. DOI: 10.1371/journal.pone.0041478

18 Zhu Z, Zhang D, Lee H, Menon AA, Wu J, Hu K and Jin Y: Macrophage-derived apoptotic bodies promote the proliferation of the recipient cells via shuttling microrna-221/222. J Leukoc Biol 101(6): 1349-1359, 2017. PMID: 28274991. DOI: 10.1189/ jlb.3A1116-483R

19 Thompson CB: Apoptosis in the pathogenesis and treatment of disease. Science 267(5203): 1456-1462, 1995. PMID: 7878464. DOI: $10.1126 /$ science. 7878464

20 Liu X, Hansen DM, Timko NJ, Zhu Z, Ames A, Qin C, Nicholl MB, Bai Q, Chen X, Wakefield MR, West G and Fang Y: Association between interleukin33 and ovarian cancer. Oncol Rep 41(2): 1045-1050, 2019. PMID: 30535474. DOI: 10.3892/ or.2018.6918
21 Zhu Z, Zhao L, Brittingham A, Bai Q, Wakefield MR and Fang Y: Trichomonas vaginalis inhibits hela cell growth through modulation of critical molecules for cell proliferation and apoptosis. Anticancer Res 38(9): 5079-5086, 2018. PMID: 30194152. DOI: 10.21873 /anticanres.12827

22 Fang Y, DeMarco VG and Nicholl MB: Resveratrol enhances radiation sensitivity in prostate cancer by inhibiting cell proliferation and promoting cell senescence and apoptosis. Cancer Sci 103(6): 1090-1098, 2012. PMID: 22417066. DOI: 10.1111/j.1349-7006.2012.02272.x

Received April 18, 2019 Revised May 23, 2019 Accepted May 28, 2019 